\title{
Otimização do tratamento ácido do yacon para inativação das polifenoloxidases com manutenção de suas propriedades funcionais
}

\author{
Optimization of the acid treatment of yacon for the inactivation of polyphenoloxidase \\ with maintenance of the functional properties
}

\author{
Nara Menezes Vieira ${ }^{1}$, Ana Paula Dionisio ${ }^{2 \star}$, Talita de Souza Goes', Idila Maria da Silva Araújo², \\ Raimundo Wilane de Figueiredo ${ }^{1}$ \\ ${ }_{1}^{1}$ Universidade Federal do Ceará (UFC), Faculdade de Engenharia de Alimentos, Fortaleza/CE - Brasil \\ 2 Embrapa Agroindústria Tropical, Fortaleza/CE - Brasil
}

\section{*Corresponding Author}

Ana Paula Dionisio, Embrapa Agroindústria Tropical, Laboratório de Processos Agroindustriais, Rua Dra. Sara Mesquita, $n^{\circ} 2270$, Planalto do Pici, CEP: 60511-110, Fortaleza/CE - Brasil, e-mail: ana.dionisio@embrapa.br

Cite as: Optimization of the acid treatment of yacon for the inactivation of polyphenoloxidase with maintenance of the functional properties. Braz. J. Food Technol., v. 21, e2016063, 2018.

Received: June 07, 2016; Accepted: Nov. 08, 2017

\section{Resumo}

O yacon é uma raiz tuberosa que se destaca por sua elevada concentração de oligossacarídeos prebióticos, principalmente os fruto-oligossacarídeos (FOS), e também pela presença de compostos fenólicos específicos, como o ácido clorogênico. Contudo, a presença destes compostos fenólicos torna o yacon susceptível a reações de escurecimento enzimático, devido à ação, principalmente, das polifenoloxidases (PPO). A ação destas enzimas pode diminuir o valor nutritivo do produto, além de afetar a sua aparência e o seu sabor, tornando-o inaceitável para o consumo. Neste sentido, o objetivo do presente trabalho foi determinar o $\mathrm{pH}$ de estabilidade e o $\mathrm{pH}$ ótimo de atividade das PPO do yacon, visando estabelecer condições que resultem em uma menor atividade desta enzima, com o uso de um tratamento ácido mais brando, uma vez que os FOS são susceptíveis à hidrólise ácida. Em posse destes resultados, realizou-se um delineamento estatístico do tipo Delineamento Central Composto Rotacional (DCCR), para avaliar o efeito de diferentes concentrações de ácido cítrico (AC) e diferentes tempos de imersão no tratamento das raízes de yacon, visando à inativação da PPO. Observou-se que o AC foi eficaz para inativação parcial da enzima PPO, sendo estatisticamente significativa $(p<0,10)$ apenas a concentração de AC (termo linear e quadrático). A porcentagem de variação explicada $\left(R^{2}\right)$ pelo modelo foi de $85,6 \%$. O tratamento selecionado pelo planejamento experimental (2,4\% de ácido cítrico por 540 segundos) ocasionou uma perda de $15 \%$ nos teores de FOS. Entretanto, o produto final apresentou $6,84 \mathrm{~g}$ FOS/100 g, muito acima do mínimo exigido pela legislação pertinente (2,5 g FOS/porção de produto) para alimentos com alegação de propriedade funcional, tornando assim viável o processo tecnológico proposto no presente trabalho.

Palavras-chave: Smallanthus sonchifolius; Fruto-oligossacarídeos; Prebióticos.

\section{Abstract}

Yacon is a tuberous root that stands out for its high concentration of prebiotic oligosaccharides, mainly fructooligosaccharides (FOS), and some specific phenolic compounds such as chlorogenic acid. However, the presence of these phenolic compounds makes yacon susceptible to enzymatic browning, due mainly to the activity of polyphenoloxidases (PPO). The action of these enzymes can decrease the nutritional value of the product, and also affect its appearance and taste, making it unacceptable for consumption. Thus the objective of the present work was to determine the optimum pH for the activity and for the stability of the yacon PPO, and establish the conditions that result in lower activity of this enzyme using a mild acid treatment, since FOS are susceptible to acid hydrolysis. Following this, a CCRD (Central Composite Rotatable Design) was used to evaluate the effect of different concentrations of citric acid (CA) and different immersion times in the treatment of yacon roots, aiming to inactivate the PPO. It was observed that CA was effective for the partial inactivation of PPO, 
Otimização do tratamento ácido do yacon para inativação das polifenoloxidases com manutenção de suas propriedades funcionais

Vieira, N. M. et al.

and that only the CA concentration (linear and quadratic terms) was statistically significant $(p<0.10)$. The percentage of variance explained $\left(R^{2}\right)$ by the model was $85.6 \%$. The treatment selected by the experimental design $(2.4 \%$ of citric acid for 540 seconds) resulted in a $15 \%$ loss of the FOS contents. However, the final product presented $6.84 \mathrm{~g} \mathrm{FOS} / 100 \mathrm{~g}$, higher than the minimum amount required by the relevant legislation ( $2.5 \mathrm{~g} \mathrm{FOS/portion} \mathrm{of} \mathrm{product)} \mathrm{for} \mathrm{a} \mathrm{food} \mathrm{to} \mathrm{claim}$ a functional property, making the technological process proposed in the present work feasible.

Keywords: Smallanthus sonchifolius; Fructooligosaccharides; Prebiotics.

\section{Introdução}

O yacon (Smallanthus sonchifolius) é uma raiz nativa dos Andes, da família Asteraceae, cujo cultivo tem se expandido a diversos países, inclusive no Brasil. O interesse nessa raiz é devido ao seu elevado teor de fruto-oligossacarídeos (FOS), que são carboidratos prebióticos capazes de estimular o desenvolvimento de bactérias benéficas no intestino humano, como bifidobactérias e lactobacilos (SANTANA; CARDOSO, 2008; DIONÍSIO et al., 2015). Além disso, o yacon apresenta características sensoriais apreciadas (sabor doce e textura crocante, semelhantes à pera e ao melão), com facilidade de incorporação em diferentes produtos alimentícios, como bebidas (DIONÍSIO et al., 2016; DIONÍSIO et al., 2015), formulações de pães (MORAIS et al., 2014), queijo tipo petit suisse (CARDARELLI et al., 2008), iogurtes (ARYANA; McGREW, 2007; CRUZ et al., 2013), dentre outros.

As raízes de yacon também contêm quantidades expressivas de compostos fenólicos, com predominância do ácido clorogênico e dos derivados do ácido cafeico (DIONÍSIO et al., 2015; CAMPOS et al., 2012; PADILHA et al., 2009). Estes compostos atuam como substrato para a ação de enzimas da classe das polifenoloxidases (PPO), com rápida formação de compostos indesejáveis (melaninas) durante o processamento desta raiz, com diminuição do valor nutritivo e alterações indesejáveis no sabor e na aparência, tornando o produto impróprio para o consumo (NEVES; SILVA, 2007; PADILHA et al., 2009).

A utilização de métodos de inativação enzimática de baixo custo que possibilitem a obtenção de um extrato de yacon com apreciáveis características sensoriais e manutenção dos seus componentes funcionais (FOS) torna-se essencial para viabilizar a incorporação do yacon em alimentos. Este controle do escurecimento enzimático pode ser feito através de métodos físicos ou químicos de conservação de alimentos (PADILHA et al., 2009), destacando-se, dentre estes, o uso de ácidos orgânicos (ARAÚJO, 2008). Porém, o tempo de imersão e a concentração do ácido podem ser considerados pontos críticos do processamento, pois, em concentrações muito elevadas $(\mathrm{pH}<4)$ e por tempo prolongado, podem ocorrer grandes perdas nos teores de FOS, devido à hidrólise ácida desses carboidratos (PASSOS; PARK, 2003), com formação de mono e dissacarídeos, como glicose, frutose e sacarose. Devido a este fato, é importante aliar a concentração de ácido utilizada com o uso de tempo relativamente curto, porém que tais condições sejam eficientes para o controle do escurecimento enzimático do yacon.

Desta forma, o objetivo do presente trabalho foi avaliar o emprego de ácido cítrico para inibição do escurecimento enzimático do yacon, utilizando um planejamento estatístico do tipo Delineamento Composto Central Rotacional (DCCR), permitindo conhecer e obter condições que garantam a incorporação desta raiz em produtos processados sem alteração na qualidade do produto final.

\section{Material e métodos}

\subsection{Material}

As raízes do yacon (Smallanthus sonchifolius) foram adquiridas no comércio local de Fortaleza-CE e processadas no Laboratório de Processos Agroindustriais, da Embrapa Agroindústria Tropical, ainda no mesmo dia.

\subsection{Caracterização da enzima PPO do yacon}

- Obtenção do extrato aquoso de yacon (EAY): Para obtenção do extrato de yacon, $100 \mathrm{~g}$ da porção comestível da raiz foi triturada em liquidificador por 60 segundos, com adição de $100 \mathrm{~mL}$ de água destilada. Após filtração em papel Whatman $n .{ }^{\circ} 1$, o material foi denominado de extrato aquoso de yacon (EAY).

- Determinação da atividade enzimática $(A E)$ : A atividade de PPO nos extratos foi determinada como descrito por Paz (2010), com modificações. Adicionou-se $0,1 \mathrm{~mL}$ de EAY à mistura de $2,9 \mathrm{~mL}$ de catecol 0,01 M/L em tampão fosfato $0,1 \mathrm{M}$, pH 6,0. Após 15 segundos, a absorbância foi lida em espectrofotômetro (Varian Cary ${ }^{\circledR} 50$ Bio - UV visível) a $420 \mathrm{~nm}$. O branco foi preparado com a mistura de 2,9 $\mathrm{mL}$ de catecol $0,01 \mathrm{M} \mathrm{e} \mathrm{0,1} \mathrm{mL}$ do tampão fosfato, $\mathrm{pH}$ 6,0. Uma unidade de atividade enzimática (AE) da PPO foi definida como a quantidade da enzima que ocasiona o aumento de 0,001 na absorbância por minuto por mililitro de amostra. 
Otimização do tratamento ácido do yacon para inativação das polifenoloxidases com manutenção de suas propriedades funcionais

Vieira, N. M. et al.

- Efeito do pH na atividade da PPO do EAY: Utilizaram-se 2,0 mL dos sistemas tampão acetato (pH 3,8 - 5,2), tampão fosfato (pH 5,8-7,8) e tampão bórax-hidróxido de sódio (pH 9,2 - 10,1), aos quais adicionaram-se 2,0 $\mathrm{mL}$ de EAY. Para a determinação da AE em cada um dos ensaios, utilizou-se $0,1 \mathrm{~mL}$ de EAY adicionado de $2,9 \mathrm{~mL}$ de solução de catecol 0,01 M. Em seguida, a $A E$ foi determinada por espectrofotômetro a $420 \mathrm{~nm}$, segundo metodologia descrita no item "Determinação da atividade enzimática (AE)".

- Efeito do pH na estabilidade da PPO do EAY: O efeito do pH na estabilidade da PPO foi determinado na faixa de $\mathrm{pH}$ de 3,8 a 10,1, utilizando

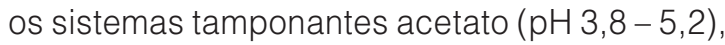
fosfato ( $\mathrm{pH} 5,8$ - 7,8) e bórax-hidróxido de sódio (pH 9,2 - 10,1). Utilizaram-se 2,0 mL de EAY adicionados em 2,0 mL de soluções tampão de diferentes valores de $\mathrm{pH}$, e incubação por 18 horas, a $25^{\circ} \mathrm{C}$. Posteriormente, a atividade residual da PPO foi determinada no $\mathrm{pH}$ ótimo de $\mathrm{AE}$, utilizando-se tampão fosfato $0,1 \mathrm{M}$ a pH 7,2. Em seguida, 0,1 mL do EAY foi adicionado a 2,9 $\mathrm{mL}$ de solução de catecol 0,01 M. Após, realizou-se leitura em espectrofotômetro a $420 \mathrm{~nm}$, segundo metodologia descrita no item "Determinação da atividade enzimática $(\mathrm{AE})$ ".

\subsection{Planejamento experimental e definição das condições de inibição do escurecimento enzimático}

Após definição do pH ótimo de atividade e de estabilidade da PPO do yacon, foi realizado um delineamento do tipo DCCR para inativação enzimática, utilizando-se o ácido cítrico. Para o delineamento estatístico $\left[2^{2}+2(2)+3 P C\right]$, utilizaram-se, como variáveis independentes, a concentração de "ácido cítrico" (- $\alpha=0,2 ;+\alpha=4,6$, em porcentagem) e o "tempo de imersão" ( $\alpha=60 ;+\alpha=540$, em segundos), conforme Tabela 1. A variável dependente foi a atividade da PPO (em U/g de EAY).

\section{- Preparo das amostras para o planejamento} estatístico: O yacon foi lavado em água corrente, descascado manualmente, sanitizado (imersão em solução clorada, 200 ppm de cloro ativo/L de água, durante 15 minutos) e cortado em cubos de cerca de $1 \mathrm{~cm}^{3}$, utilizando-se cortador de legumes. Em seguida, foi submetido ao tratamento com soluções acidificadas, de acordo com o planejamento experimental. No final de cada um dos processamentos, as amostras foram trituradas em liquidificador durante 60 segundos (temperatura ambiente, $25 \pm 2{ }^{\circ} \mathrm{C}$ ), com adição
Tabela 1. Valores utilizados no Delineamento Central Composto Rotacional (DCCR).

\begin{tabular}{lccccc}
\multicolumn{1}{c}{ Variáveis } & \multicolumn{5}{c}{ Nível } \\
\cline { 2 - 6 } & $\mathbf{- 1 , 4 1}$ & $\mathbf{- 1}$ & $\mathbf{0}$ & $\mathbf{+ 1}$ & $\mathbf{+ 1 , 4 1}$ \\
Ácido cítrico (\%) & 0,2 & 0,8 & 2,4 & 4,0 & 4,6 \\
$\begin{array}{l}\text { Tempo de imersão } \\
\text { (segundos) }\end{array}$ & 60 & 130 & 300 & 470 & 540 \\
\hline
\end{tabular}

de água $\left(25 \pm 2{ }^{\circ} \mathrm{C}\right)$, na proporção 1:1 (m/v), e embaladas em recipientes de plástico contendo $60 \mathrm{~g}$ de amostra cada. Após o processamento, as amostras permaneceram em temperatura ambiente $\left(25 \pm 2{ }^{\circ} \mathrm{C}\right)$ por 120 minutos e foram então analisadas quanto à atividade da PPO. Os resultados foram avaliados através do programa Protimiza Experimental Design (PROTIMIZA, 2014), considerando grau de significância de $10 \%$.

- Caracterização das amostras selecionadas pelo planejamento estatístico: Após definição das condições ótimas de processo, os FOS e os mono e dissacarídeos (glicose, frutose e sacarose) foram determinados na amostra tratada na condição otimizada e na amostra controle (sem tratamento). Todas as análises foram realizadas em triplicata.

- Determinação de fruto-oligossacarídeos (FOS): Os FOS foram determinados segundo metodologia descrita pela AOAC (2010) e expressos em $\mathrm{g} / 100 \mathrm{~g}$ de yacon.

- Determinação de mono e dissacarídeos por CLAE - IR: Os mono e dissacarídeos (glicose, frutose e sacarose) foram determinados nas amostras, segundo metodologia descrita por Burgner e Feinberg, 1992. Resumidamente, $10 \mathrm{~mL}$ de amostra foram diluídos com água deionizada, na proporção de 1:5 (v/v). Posteriormente, foram adicionados $3 \mathrm{~mL}$ de uma solução de ferrocianeto de potássio $(0,25 \mathrm{M})$ e 3,5 $\mathrm{mL}$ de solução de acetato de zinco $(1,0 \mathrm{M})$, sendo o volume aferido até $100 \mathrm{~mL}$, em balão volumétrico. A solução de amostra foi filtrada, utilizando-se papel filtro Whatman $n .{ }^{\circ} 1$ e, em seguida, utilizou-se membrana filtrante de PVDF de 0,22 $\mu \mathrm{m}$ e $13 \mathrm{~mm}$ de diâmetro (Millipore). As condições cromatográficas foram: detector de índice de refração Varian, modelo ProStar 350, ajustado em $35^{\circ} \mathrm{C}$; coluna Zorbax Carbohydrate $(250 \times 4,6 \mathrm{~mm}, 5 \mu \mathrm{m}$, Agilent); temperatura da coluna de $35{ }^{\circ} \mathrm{C}$; fase móvel composta de acetonitrila e água deionizada (80:20, v/v), e vazão da fase móvel de $1,5 \mathrm{~mL} / \mathrm{min}$, mantida constante até o final da análise. Os resultados foram avaliados por Análise de Variância e as médias foram comparadas pelo Teste de Tukey $(\alpha=0,05)$, usando-se o programa SAS para Windows (SAS INSTITUTE, 2009). 
Otimização do tratamento ácido do yacon para inativação das polifenoloxidases com manutenção de suas propriedades funcionais

Vieira, N. M. et al.

\section{Resultados e discussão}

\section{$3.10 \mathrm{pH}$ ótimo de atividade e o pH de estabilidade da polifenoloxidase (PPO) do extrato aquoso de yacon (EYA)}

A Tabela 2 apresenta os resultados do $\mathrm{pH}$ ótimo de atividade e o pH de estabilidade da PPO do extrato aquoso de yacon (EYA), frente aos distintos tratamentos.

A PPO do yacon apresentou atividade máxima quando o pH foi ajustado em 7,2 e 7,8, com $A E=343,6 \pm 4,5$ e $324,4 \pm 9,6 \mathrm{U} / \mathrm{g}$ de EAY, respectivamente. Nestas condições, não houve diferença estatisticamente significativa $(p<0,05)$ entre os tratamentos. Para os dois extremos de $\mathrm{pH}$ avaliados ( $\mathrm{pH} 3,8$ e 10,1), os valores de AE foram de 140,7 a 69,5 U/g (Tabela 2). As plantas, em geral, apresentam atividade máxima da PPO em pH neutro ou próximo da neutralidade, sendo comum a atividade máxima na faixa de pH de 5,0 a 7,0 (GAWLIK-DZIKI et al., 2008). Diversos autores avaliaram o pH ótimo de atividade da PPO utilizando diferentes alimentos. Observe-se que o estudo da atividade enzimática para cada alimento é de extrema importância, visto que o pH ótimo da PPO varia de acordo com a fonte da enzima e depende também do substrato fenólico (NEVES; SILVA, 2007). Em batatas, Duangmal e Apenten (1999) relataram que a faixa ótima de $\mathrm{pH}$ de atividade da PPO é de 6,6 a 7,2, sendo a atividade reduzida para menos de $40 \%$ quando o $\mathrm{pH}$ encontra-se abaixo de 6,0. Para yacon, utilizando-se PPO parcialmente purificada e diferentes substratos fenólicos, a atividade ótima da enzima variou de 5,0 a 6,6 (NEVES; SILVA, 2007). Rodrigues et al. (2014), por sua vez, trabalhando com yacon, reportaram que o $\mathrm{pH}$ de atividade ótima para a PPO foi de 7,0, utilizando o catecol como substrato. Embora os resultados encontrados apresentem variações inerentes aos vegetais analisados e aos substratos fenólicos utilizados,

Tabela 2. O pH ótimo de atividade enzimática (AE) e a estabilidade da PPO de extrato aquoso de yacon (EAY).

\begin{tabular}{ccc}
$\begin{array}{c}\text { Tratamento } \\
(\mathbf{p H})\end{array}$ & $\begin{array}{c}\mathbf{A E} \\
\mathbf{( U / g} \text { de EAY) }\end{array}$ & $\begin{array}{c}\text { AE remanescente } \\
\text { após tratamento } \\
(\%)\end{array}$ \\
\hline 3,8 & $140,7 \pm 2,3^{\mathrm{b}}$ & $16,2 \pm 0,7^{\mathrm{d}}$ \\
4,2 & $133,0^{\mathrm{d}} \pm 5,2^{\mathrm{bc}}$ & $17,9 \pm 0,8^{\mathrm{d}}$ \\
4,8 & $143,2^{\mathrm{a}} \pm 1,2^{\mathrm{b}}$ & $49,3 \pm 2,2^{\mathrm{bcd}}$ \\
5,2 & $113,9 \pm 11,8^{\mathrm{c}}$ & $40,7 \pm 0,7^{\mathrm{cd}}$ \\
5,8 & $72,9 \pm 10,9^{\mathrm{de}}$ & $46,1 \pm 3,9^{\mathrm{bcd}}$ \\
6,2 & $85,25 \pm 12,9^{\mathrm{d}}$ & $73,4 \pm 1,6^{\mathrm{abc}}$ \\
6,8 & $145,6 \pm 1,4^{\mathrm{b}}$ & $79,1 \pm 1,2^{\mathrm{ab}}$ \\
7,2 & $343,6 \pm 4,5^{\mathrm{a}}$ & $81,4 \pm 1,1^{\mathrm{ab}}$ \\
7,8 & $324,4 \pm 9,6^{\mathrm{a}}$ & $100,0 \pm 2,2^{\mathrm{a}}$ \\
9,2 & $86,2 \pm 3,8^{\mathrm{d}}$ & $61,1 \pm 0,3^{\mathrm{bc}}$ \\
9,8 & $63,2 \pm 12,0^{\mathrm{e}}$ & $40,0 \pm 7,6^{\mathrm{cd}}$ \\
10,1 & $69,5 \pm 8,0^{\mathrm{de}}$ & $42,8 \pm 6,4^{\mathrm{cd}}$ \\
\hline
\end{tabular}

Números seguidos pelas mesmas letras em cada coluna não diferem significativamente no nível de 5\% pelo Teste de Tukey. esses resultados mostram que os valores ótimos de $\mathrm{pH}$ encontrados para atividade máxima da enzima PPO estão de acordo com a literatura.

Com relação à estabilidade da enzima frente a diferentes condições de $\mathrm{pH}$, verificou-se que PPO do yacon ficou estável na faixa de pH de 6,2 a 7,8 (Tabela 2), mantendo mais de $70 \%$ da atividade inicial, após 18 horas, a $25{ }^{\circ} \mathrm{C}$. Nestas condições, não houve diferenças estatisticamente significativas entre estes tratamentos $(p<0,05)$. Além disso, a PPO do yacon mostrou menor estabilidade em pH inferior a 4,2, em que apresentou cerca de $18 \%$ da atividade inicial da enzima, chegando a $16 \%$ em pH 3,8. Porém, os FOS são relativamente instáveis em pH inferiores a 4,0 (PASSOS; PARK, 2003), sendo um fator limitante trabalhar em condições ainda mais ácidas, para completa inativação da enzima. Resultado semelhante foi obtido em um estudo com hortelã-pimenta, em que a enzima ainda apresentou $20 \%$ de atividade em pH 4,0 (KAVRAYAN; AYDEMIR, 2001). Em estudo com a PPO da uva, os autores verificaram que, em $\mathrm{pH}$ ácido (faixa de 2,5 - 4,0), a PPO ainda apresentou atividade, pois, em $\mathrm{pH} 3,5$, a enzima apresentou $70 \%$ de atividade residual, e em pH alcalino (faixa de 7,0-9,0) a atividade da enzima diminuiu rapidamente, apresentando apenas $20 \%$ de atividade em pH 8,0 (RAPEANU et al., 2006). Note-se que, no presente trabalho, uma redução de apenas $40 \%$ e $43 \%$ foi verificada em $\mathrm{pH}$ de 9,8 e 10,2, respectivamente.

\subsection{Otimização do uso de ácido cítrico (AC) para inativação da polifenoloxidase (PPO) do yacon}

Em posse dos resultados de $\mathrm{pH}$ ótimo de atividade e pH de estabilidade, verificou-se que a PPO apresenta menor estabilidade em condições ácidas, especialmente aquelas inferiores a $4,2 \%$. Isso fornece indicativos de que o uso de um acidulante, em concentrações apropriadas, pode afetar diretamente a atividade e a estabilidade da enzima, sendo interessante o seu uso em processos que visem ao controle do escurecimento ocasionado pela ação da PPO.

De uma forma geral, os inibidores da atividade da PPO podem ser divididos em seis categorias, sendo estas: (a) agentes redutores (ácido ascórbico, por exemplo); (b) acidulantes (ácidos cítrico e fosfórico); (c) agentes quelantes (como o EDTA); (d) agentes complexantes (ciclodextrina); (e) inibidores de enzimas (como ácidos carboxílicos aromáticos, por exemplo), ou (f) uso de enzimas específicas (como oxigenases ou proteases) (GARZÓN et al., 2011). Dentre os acidulantes, o ácido cítrico tem sido extensivamente utilizado para evitar o escurecimento enzimático de diversos produtos alimentares, devido ao seu relativo baixo custo, à facilidade de emprego e ao amplo espectro de uso. Além da diminuição do pH, o efeito inibitório do ácido cítrico também ocorre devido 
Otimização do tratamento ácido do yacon para inativação das polifenoloxidases com manutenção de suas propriedades funcionais

Vieira, N. M. et al.

à quelação do cobre localizado no sítio ativo da PPO (HOLZWARTH et al., 2012).

Neste sentido, o ácido cítrico foi utilizado para a inativação da PPO em um planejamento estatístico do tipo DCCR, no qual foi avaliada a influência da "concentração do ácido" e do "tempo de imersão em solução ácida" (variáveis independentes) na atividade da PPO (variável dependente). As condições experimentais realizadas e seus respectivos resultados em termos de AE estão apresentados na Tabela 3.

Através das análises dos coeficientes de regressão (Tabela 4), pode-se visualizar que apenas os termos linear e quadrático da concentração de AC foram significativos $(p<0,10)$, e que a porcentagem de variação explicada pelo modelo foi de $85,6 \%$. Os demais termos foram incorporados aos resíduos para cálculo da ANOVA (Tabela 5).

Os resultados do planejamento estatístico demonstraram que, de uma forma geral, apenas o ácido cítrico contribuiu $(p<0,10)$ para uma menor atividade da PPO. O tempo de imersão estudado, que variou de 60 a 540 segundos, não teve influência na atividade da enzima. De acordo com a Figura 1, é possível verificar que a faixa ótima é de $2,4 \%$ a 3,9\%. Embora o tempo de permanência na solução ácida não tenha sido significativo, optou-se por considerar os valores obtidos pela regressão sem a incorporação dos termos não significativos ao cálculo dos resíduos. Desta forma, a condição ótima de tempo de permanência em solução seria de aproximadamente 540 segundos.

Rodrigues et al. (2014), avaliando o uso de uma combinação de agentes químicos para inibição da PPO de farinha de yacon, verificaram que o uso de ácido cítrico, L-cisteína e ácido ascórbico foi eficiente para a inativação enzimática. Através de um planejamento também do tipo DCCR, os autores verificaram que todos os ácidos, tanto os termos lineares como os quadráticos, assim como suas interações, foram significativas para o modelo $(p<0,05)$.

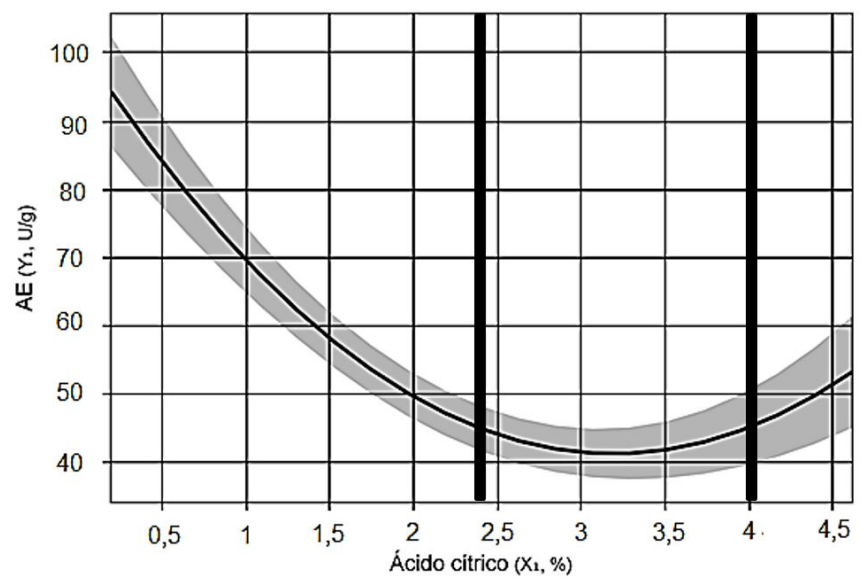

Figura 1. Curva de contorno para ácido cítrico (\%), com resposta de atividade enzimática da PPO (AE, em U/g).
Porém, o tempo de imersão foi padronizado para todos os tratamentos (20 minutos), não sendo uma variável do planejamento estatístico.

No presente trabalho, o tratamento, na condição definida pelo planejamento experimental, foi submetido à quantificação dos teores de FOS, assim como de glicose, frutose e sacarose (Tabela 6).

Com relação aos mono e dissacarídeos, verificou-se uma diminuição significativa $(p<0,05)$ nos valores de glicose e frutose, assim como um aumento dos valores de sacarose. A degradação do FOS provém da despolimerização das

Tabela 3. Matriz do planejamento experimental completo (valores codificados) dos tratamentos com ácido cítrico, com a resposta da atividade enzimática (AE) da PPO.

\begin{tabular}{cccc} 
Ensaios & $\begin{array}{c}\text { Ácido cítrico } \\
(\%)\end{array}$ & $\begin{array}{c}\text { Tempo de } \\
\text { imersão } \\
\text { (segundos) }\end{array}$ & $\begin{array}{c}\text { AE } \\
\mathbf{( U / g )}\end{array}$ \\
\hline 1 & $-1(0,8)$ & $-1(130)$ & 61,5 \\
2 & $+1(4,0)$ & $-1(130)$ & 51,9 \\
3 & $-1(4,0)$ & $+1(470)$ & 65,3 \\
4 & $+1(0,8)$ & $+1(470)$ & 42,6 \\
5 & $-1,41(0,2)$ & $0(240)$ & 107,0 \\
6 & $+1,41(4,6)$ & $0(240)$ & 47,7 \\
7 & $0(2,4)$ & $-1,41(60)$ & 46,7 \\
8 & $0(2,4)$ & $+1,41(540)$ & 42,4 \\
9 & $0(2,4)$ & $0(240)$ & 47,0 \\
10 & $0(2,4)$ & $0(240)$ & 47,4 \\
11 & $0(2,4)$ & $0(240)$ & 47,8 \\
\hline
\end{tabular}

Tabela 4. Coeficientes de regressão do DCCR para a atividade da PPO.

\begin{tabular}{ccccc} 
& $\begin{array}{c}\text { Coeficientes } \\
\text { de } \\
\text { regressão }\end{array}$ & $\begin{array}{c}\text { Erro } \\
\text { padrão }\end{array}$ & $\begin{array}{c}\mathbf{t} \\
\text { calculado }\end{array}$ & $\boldsymbol{p}$-valor \\
\hline Média & 47,39 & 5,14 & 9,22 & 0,0002 \\
$\mathrm{x}_{1}$ & $-14,52$ & 3,14 & $-4,61$ & 0,0057 \\
$\mathrm{x}_{1}^{2}$ & 13,56 & 3,74 & 3,62 & 0,0151 \\
$\mathrm{x}_{2}$ & $-1,44$ & 3,14 & $-0,46$ & 0,6647 \\
$\mathrm{x}_{2}^{2}$ & $-2,83$ & 3,74 & $-0,75$ & 0,4837 \\
$\mathrm{x}_{1} \cdot \mathrm{x}_{2}$ & $-3,27$ & 4,44 & $-0,73$ & 0,4947 \\
\hline $\mathrm{x}_{1}=\mathrm{m}_{2}$ & & &
\end{tabular}

$\mathrm{x}_{1}=$ ácido cítrico (em porcentagem) e $\mathrm{x}_{2}=$ tempo de imersão (em segundos)

Tabela 5. ANOVA para a atividade da PPO.

\begin{tabular}{lcccccc}
$\begin{array}{l}\text { Fonte de } \\
\text { variação }\end{array}$ & $\begin{array}{c}\text { Soma dos } \\
\text { quadrados }\end{array}$ & GL* $^{*} \begin{array}{c}\text { Quadrados } \\
\text { médios }\end{array}$ & $\mathbf{F}_{\text {calc }}$ & p-valor \\
\hline \multicolumn{7}{c}{ Tratamento com ácido cítrico } \\
\hline Regressão & 2968,85 & 2 & 1484,43 & $23,71<0,001$ \\
Resíduos & 500,92 & 8 & 62,62 & & \\
Total & 3469,77 & 10 & 346,97 & \\
\hline
\end{tabular}

Variação explicada pelo modelo foi de $85,6 \%$. $F_{\text {tab }}(2 ; 8 ; 0,10)=4,46$. ${ }^{*} \mathrm{GL}=$ Grau de Liberdade. 
Otimização do tratamento ácido do yacon para inativação das polifenoloxidases com manutenção de suas propriedades funcionais

Vieira, N. M. et al.

Tabela 6. Teor de FOS, mono e dissacarídeos no yacon sem tratamento (controle) e no yacon após o tratamento com 2,4\% de ácido cítrico, por 540 segundos (média e desvio padrão).

\begin{tabular}{|c|c|c|c|c|}
\hline Tratamento & $\begin{array}{c}\text { FOS } \\
(g / 100 \mathrm{~g})\end{array}$ & $\begin{array}{l}\text { Glicose } \\
(\mathrm{g} / 100 \mathrm{~g})\end{array}$ & $\begin{array}{l}\text { Frutose } \\
(\mathrm{g} / 100 \mathrm{~g})\end{array}$ & $\begin{array}{l}\text { Sacarose } \\
(\mathrm{g} / 100 \mathrm{~g})\end{array}$ \\
\hline Controle & $8,13 \pm 0,16^{a}$ & $0,98 \pm 0,02^{a}$ & $0,70 \pm 0,02^{a}$ & $0,82 \pm 0,02^{a}$ \\
\hline Ácido cítrico & $6,84 \pm 0,09^{b}$ & $0,91 \pm 0,01^{b}$ & $0,44 \pm 0,01^{b}$ & $1,40 \pm 0,05^{b}$ \\
\hline
\end{tabular}

Números seguidos pelas mesmas letras em cada coluna não diferem significativamente no nível de $5 \%$ pelo Teste de Tukey.

cadeias dessas fibras pela ação do ácido, que rompe a cadeia entre os resíduos de frutose até a molécula terminal de sacarose. Desta forma, o aumento da concentração de sacarose é confirmado pela diminuição significativa da concentração de FOS ( $p<0,05)$, quando comparado ao tratamento controle, com perda de aproximadamente $15 \%$ de FOS. Estes valores são inferiores aos reportados por Fante et al. (2013), que estudaram a inibição da PPO com uso de calor. Os autores reportaram uma diminuição de $84 \%$ na atividade da PPO, quando a raiz tuberosa foi submetida ao tratamento térmico de $100^{\circ} \mathrm{C}$, por 4 minutos. Porém, embora a atividade enzimática tenha sido reduzida, as perdas de FOS foram cerca de $30 \%$, nesta condição avaliada. Matusek et al. (2009), avaliando diferentes condições de $\mathrm{pH}$ e temperatura na estabilidade de alguns oligossacarídeos (dentre os quais, os FOS), demonstraram que a hidrólise ácida dos FOS é insignificante em temperaturas até $60^{\circ} \mathrm{C}$. Entretanto, quase a totalidade destes compostos é degradada em temperatura de $90{ }^{\circ} \mathrm{C}$ a $100{ }^{\circ} \mathrm{C}$, por um período de $1 \mathrm{~h}$ a $1,5 \mathrm{~h}$, mostrando claramente um forte efeito da temperatura na degradação do FOS.

A legislação vigente para produtos com alegações de propriedades funcionais considera que a porção do produto deva conter um mínimo de 2,5 g de fruto-oligossacarídeos, e a alegação permitida é "os fruto-oligossacarídeos - FOS (prebiótico) contribuem para o equilíbrio da flora intestinal. Seu consumo deve estar associado a uma alimentação equilibrada e hábitos de vida saudáveis" (BRASIL, 2008). Assim, pode-se observar que a concentração de FOS obtida ao final do processamento ácido (6,84 g de FOS/100 g) está muito acima do mínimo exigido pela legislação sanitária brasileira (BRASIL, 2008). Desta forma, o processo tecnológico proposto no presente trabalho torna-se uma estratégia viável, uma vez que se usa um ácido comumente utilizado pela indústria de alimentos e de relativo baixo custo. Além disso, o processo otimizado ocasiona somente $15 \%$ de perda de FOS durante o processamento, tornando o produto final rico nestes compostos de interesse e permitindo a sua incorporação em alimentos funcionais.

\section{Conclusões}

A polifenoloxidase do yacon apresentou atividade máxima em $\mathrm{pH}$ 7,2 e mostrou-se estável na faixa de $\mathrm{pH}$ de 6,2 a 7,8 , mantendo mais de $70 \%$ da atividade inicial após
18 horas, a $25^{\circ} \mathrm{C}$. Através do delineamento estatístico, foi possível propor condições de processo para inativação desta enzima utilizando ácido cítrico, com manutenção dos fruto-oligossacarídeos (FOS) do yacon. A condição otimizada pelo planejamento estatístico, com imersão da raiz tuberosa por 540 segundos em uma solução a 2,4\% de ácido cítrico, ocasionou uma perda de 15\% nos teores de FOS. O produto final apresentou 6,84 g FOS/100 g, tornando viável o processo tecnológico proposto no presente trabalho e permitindo a sua incorporação em alimentos funcionais.

\section{Agradecimentos}

Os autores agradecem à Embrapa Agroindústria Tropical e ao CNPq pelo apoio financeiro.

\section{Referências}

ARAÚJO, J. M. A. Química de alimentos: teoria e prática. 4. ed. Viçosa: UFV, 2008. 596 p

ARYANA, K. J.; MCGREW, P. Quality attributes of yogurt with Lactobacillus casei and various prebiotics. Food Science and Technology, v. 40, p. 1008-1014, 2007

ASSOCIATION OF OFFICIAL ANALYTICAL CHEMISTS - AOAC. Chapter 45: method 999.03. In: HORWITZ, W.; LATIMER, J. R.; GEORGE, W. (Ed.). Official methods of analysis of the Association of Official Analytical Chemists. 18th ed. Gaithersburg: AOAC, 2010. p. 96-98.

BRASIL. Alimentos com alegações de propriedades funcionais e ou de saúde, novos alimentos/ingredientes, substâncias bioativas e probióticos - lista de alegações de propriedade funcional aprovadas. Brasília: Anvisa, 2008. Disponível em: $<$ http://portal.anvisa.gov.br/alimentos/alegacoes>. Acesso em: 05 jun. 2016

BURGNER, E.; FEINBERG, M. Determination of mono- and disaccharides in foods by interlaboratory study: quantitation of bias components for liquid chromatography. Journal of AOAC International, v. 75, p. 443-464, 1992.

CAMPOS, D.; BETALLELUZ-PALLARDEL, I.; CHIRINOS, R.; AGUILAR-GALVEZ, A.; NORATTO, G.; PEDRESCHI, R. Prebiotic effects of yacon (Smallanthus sonchifolius Poepp. \& Endl), a source of fructooligosaccharides and phenolic compounds with antioxidant activity. Food Chemistry, v. 135, n. 3, p. 
Otimização do tratamento ácido do yacon para inativação das polifenoloxidases com manutenção de suas propriedades funcionais

Vieira, N. M. et al.

1592-1599, 2012. PMid:22953898. http://dx.doi.org/10.1016/j. foodchem.2012.05.088.

CARDARELLI, H. R.; BURITI, F. C. A.; CASTRO, I. A.; SAAD, S. M. I. Inulin and oligofructose improve sensory quality and increase the probiotic viable count in potentially synbiotic petitsuisse cheese. Food Science and Technology, v. 41, n. 6, p. 1037-1046, 2008.

CRUZ, A. G.; CAVALCANTI, R. N.; GUERREIRO, L. M. R.; SANT'ANA, A. S.; NOGUEIRA, L. C.; OLIVEIRA, C. A. F.; DELIZA, R.; CUNHA, R. L.; FARIA, J. A. F.; BOLINI, H. M. A. Developing a prebiotic yogurt: rheological, physico-chemical and microbiological aspects and adequacy of survival analysis methodology. Journal of Food Engineering, v. 114, n. 3, p. 323-330, 2013. http:// dx.doi.org/10.1016/j.jfoodeng.2012.08.018.

DIONÍSIO, A. P.; CARVALHO-SILVA, L. B.; VIEIRA, N. M.; GOES, T. S.; WURLITZER, N. J.; BORGES, M. F.; BRITO, E. S.; IONTA, M.; FIGUEIREDO, R. W. Cashew-apple (Anacardium occidentale L.) and yacon (Smallanthus sonchifolius) functional beverage improve the diabetic state in rats. Food Research International, v. 177, p. 171-176, 2015. http://dx.doi.org/10.1016/j.foodres.2015.07.020.

DIONíSIO, A. P.; WURLITZER, N. J.; GOES, T. S.; BORGES, M. F.; GARRUTI, D. S.; ARAUJO, I. M. S. Estabilidade de uma bebida funcional de frutas tropicais e yacon (Smallanthus sonchifolius) durante o armazenamento sob refrigeração. Archivos Latinoamericanos de Nutricion, v. 66, p. 148, 2016.

DUANGMAL, K.; APENTEN, R. K. O. A comparative study of polyphenoloxidases from taro (Colocasia esculenta) and potato (Solanum tuberosum var. Romano). Food Chemistry, v. 64, n. 3, p. 351-359, 1999. http://dx.doi.org/10.1016/S0308-8146(98)00127-7.

FANTE, L.; SCHER, C. F.; NOREÑA, C. P. Z.; RIOS, A. O. Study of enzyme inactivation using steam in yacon (Smallanthus sonchifolius) roots. Journal of Food Processing and Preservation, v. 37, n. 1, p. 16-24, 2013. http://dx.doi.org/10.1111/j.17454549.2011.00609.x.

GARZÓN, M. A. G.; ROJANO, B. A.; GUERRERO, C. A. Inhibición de la polifenoloxidasa extraída del banano (cavendish) por medio de algunos derivados del isoespintanol. In: GARZÓN, M. A. G.; ROJANO, B. A.; GUERRERO, C. A. Lasallista, investigacion y ciencia. Antioquia: Corporacion Universitaria Lasallista, 2011. p. $193-247$

GAWLIK-DZIKI, U.; ZŁOTEK, U.; ŚWIECA, M. Characterization of polyphenol oxidase from butter lettuce (Lactuca sativavar capitata L.). Food Chemistry, v. 107, n. 1, p. 129-135, 2008. PMid:26054273. http://dx.doi.org/10.1016/j.foodchem.2007.07.068.

HOLZWARTH, M.; WITTIG, J.; CARLE, R.; KAMMERER, D. R. Influence of putative polyphenoloxidase (PPO) inhibitors on strawberry (Fragaria $x$ ananassa Duch.) PPO, anthocyanin and color stability of stored purées. Lebensmittel-Wissenschaft + Technologie, v. 52, n. 2, p. 1-12, 2012.
KAVRAYAN, D.; AYDEMIR, T. Partial purification and characterization of polyphenoloxidase from peppermint (Mentha piperita). Food Chemistry, v. 74, n. 2, p. 147-154, 2001. http://dx.doi.org/10.1016/ S0308-8146(01)00106-6.

MATUSEK, A.; MERÉSZ, P.; LE, T. K. D.; ÖRSI, F. Effect of temperature and $\mathrm{pH}$ on the degradation of fructo-oligosaccharides. European Food Research and Technology, v. 228, n. 3, p. 355-365, 2009. http://dx.doi.org/10.1007/s00217-008-0941-8.

MORAIS, E. C.; CRUZ, A. G.; FARIA, J. A. F.; BOLINI, H. M. A. Prebiotic gluten-free bread: sensory profiling and drivers of liking. Food Science and Technology, v. 55, n. 1, p. 248-254, 2014.

NEVES, V. A.; SILVA, M. A. Polyphenol oxidase from yacon roots (Smallanthus sonchifolius). Journal of Agricultural and Food Chemistry, v. 55, n. 6, p. 2424-2430, 2007. PMid:17316020. http://dx.doi.org/10.1021/jf063148w.

PADILHA, V. M.; ROLIM, P. M.; SALGADO, S. M.; LIVERA, A. V. S.; OLIVEIRA, M. G. Tempo de secagem e da atividade de oxidoredutases de yacon (Smallanthus sonchifolius) sob tratamento químico. Ciência Rural, v. 39, n. 7, p. 2178-2184, 2009. http:// dx.doi.org/10.1590/S0103-84782009005000142.

PASSOS, L. M. L.; PARK, Y. K. Frutooligossacarídeos: implicações na saúde humana e utilização em alimentos. Ciência Rural, v. 33, n. 2, p. 385-390, 2003. http://dx.doi.org/10.1590/S010384782003000200034 .

PAZ, J. C. S. N. Caracterização bioquímica da polifenoloxidase e da peroxidase de ameixa rubimel, polpa de cacau e estudo do efeito de agentes anti-escurecimento. 2010. 85 f. Tese (Pós-graduação em Ciência de Alimentos)--Universidade Estadual de Campinas, Campinas, 2010.

PROTIMIZA. Protimiza experimental design. Campinas: PROTIMIZA, 2014. Versão 1. Disponível em: <experimentaldesign.protimiza.com.br>. Acesso em: 05 jun. 2016.

RAPEANU, G.; LOEY, A. V.; SMOUT, C.; HENDRICKX, M. Biochemical characterization and process stability of polyphenoloxidase extracted from Victoria grape (Vitis vinifera ssp. Sativa). Food Chemistry, v. 94, n. 2, p. 253-261, 2006. http://dx.doi.org/10.1016/j. foodchem.2004.10.058.

RODRIGUES, O. R. L.; ASQUIERI, E. R.; ORSI, D. C. Prevention of enzymatic browning of yacon flour by the combined use of anti-browning agents and the study of its chemical composition. Food Science and Technology, v. 34, n. 2, p. 275-280, 2014. http://dx.doi.org/10.1590/fst.2014.0045.

SANTANA, I.; CARDOSO, M. H. Raiz tuberosa de yacon (Smallanthus sonchifolius): potencialidade de cultivo, aspectos tecnológicos e nutricionais. Ciência Rural, v. 38, n. 3, p. 898-905, 2008. http:// dx.doi.org/10.1590/S0103-84782008000300050.

SAS INSTITUTE. Statistical analysis system user's guide. Cary: SAS Institute, 2009. 